\title{
MyD88-independent activation of a novel actin-Cdc42/Rac pathway is required for Toll-like receptor-stimulated phago- cytosis
}

\author{
Ling Kong ${ }^{1,2}$, Bao-Xue Ge ${ }^{1}$ \\ ${ }^{1}$ Institute of Health Science, Shanghai Institutes for Biological Sciences, Chinese Academy of Sciences and Shanghai Jiao-Tong Uni- \\ versity School of Medicine, Shanghai 200032, China; ${ }^{2}$ Graduate School of Chinese Academy of Sciences, Shanghai 200032, China
}

Phagocytosis and subsequent degradation of pathogens by macrophages play a pivotal role in host innate immune responses to microbial infection. Recent studies have shown that Toll-like receptors (TLRs) play an important role in promoting the clearance of bacteria by up-regulating the phagocytic activity of macrophages. However, information regarding the signaling mechanism of TLR-mediated phagocytosis is still limited. Here, we provide evidence that the stimulation of TLR4 with LPS leads to activation of multiple signaling pathways including MAP kinases, phosphatidylinositide 3-kinase (PI3K), and small GTPases in the murine macrophage-like cell line RAW264.7. Specific inhibition of Cdc42/Rac or p38 MAP kinase, but not PI3K, reduced TLR4-induced phagocytosis of bacteria. Moreover, we have found that either inhibition of actin polymerization by cytochalasin D or the knockdown of actin by RNAi markedly reduced the activation of Cdc42 and Rac by LPS. TLR4-induced activation of Cdc42 and Rac appears to be independent of MyD88. Taken together, our results described a novel actin-Cdc42/Rac pathway through which TLRs can specifically provoke phagocytosis.

Keywords: innate immunity, Toll-like receptors, phagocytosis, GTPases, actin, p38

Cell Research (2008) 18:745-755. doi: 10.1038/cr.2008.65; published online 10 June 2008

\section{Introduction}

Phagocytosis and subsequent degradation of pathogens by macrophages play a pivotal role in host innate immune responses to microbial infection [1]. Pathogens are detected by macrophages through a variety of phagocytic receptors such as Fc gamma receptors (Fc $\gamma \mathrm{Rs}$ ) and complement receptor 3(CR3) [2]. Ligation of these receptors activates a series of intracellular signaling pathways that lead to the dynamic and rapid reorganization of the actin cytoskeleton, which is essential for phagocytic uptake. The phagocytic process is complicated and regulated by a variety of participants, including receptors, protein kinases, effector proteins, cytoskeletal rearrangement, and Rho GTPases. The Rho GTPases Rac1 and Cdc42 play essential roles

Correspondence: Bao-Xue Ge

Tel/Fax: +86-21-63852784

E-mail: gebaoxue@sibs.ac.cn

Received 14 January 2008; revised 26 February 2008; accepted 24 March 2008; published online 10 June 2008 in actin assembly during phagocytosis [3, 4]. Inhibition of either enzyme in macrophages results in a complete blockade of FcR-mediated phagocytosis because of defective actin assembly at nascent phagosomes $[3,4]$. The activation of Cdc42 and Rac1 involves the transition from an inactive GDP-bound form to an active GTP-bound form, and is catalyzed by guanine nucleotide-exchange factors (GEFs) [5].

In addition to the phagocytic process, activation of macrophages by a variety of molecular patterns specific for microbial pathogens is mediated through Toll-like receptors (TLRs) [6]. TLRs are a family of conserved sensors of infectious nonself, and function as receptors for the activation of the innate immune system [7]. Ligation of TLRs by bacteria or their components triggers the activation of signaling cascades, leading to the production of proinflammatory cytokines. Lipopolysaccharide (LPS) is an outer membrane component of gram-negative bacteria that can be recognized by TLR4 [8]. Recognition of LPS by TLR4 recruits adaptor molecules such as myeloid differentiation factor 88 (MyD88), interleukin (IL)-1 receptor-associated kinase (IRAK), and tumor necrosis factor (TNF) receptor- 
associated factor 6 (TRAF6). Recruitment of these adaptors triggers, in turn, the activation of multiple intracellular cascades, including extracellular signal-regulated kinases (ERKs), c-Jun N-terminal kinases (JNKs), p38, and NF$\kappa \mathrm{B}$. In addition to activating MAPKs and NF- $\kappa \mathrm{B}$, LPS stimulation induces activation of the phosphatidylinositide 3-kinase (PI3K) pathway, although the molecular mechanisms by which LPS activates PI3K remain to be elucidated. Activation of these signaling pathways leads to the biosynthesis of a group of immunoregulatory molecules such as TNF, IL-12, and arachidonic acid metabolites [6, $9,10]$. These mediators are essential for the recruitment and activation of immunocompetent cells that cooperate in fighting bacterial infection.

Recent studies have shown that TLRs play an important role in promoting the clearance of bacteria by up-regulating the phagocytic activity of macrophages. Upon phagocytosis of microbial pathogens, surface TLRs, including TLR4 and TLR2, are recruited to the phagosome and become activated by microbial cell wall components $[11,12]$. Even though it is still an open question whether stimulation of TLR2 or 4 regulates phagosome maturation, ligation of TLRs has been shown to trigger MyD88-dependent signaling through IRAK-4 and p38, leading to the up-regulation of a number of phagocytic gene expression programs [13-15]. More recent studies have found that TLR2 is involved in the phagocytosis of amyloid $\beta$ peptide by microglia [16], a phagocyte specifically located in the brain, and of fungi by macrophages [17], and that TLR4 is involved in the phagocytosis of bacteria by enterocytes [18]. These results suggest that TLR participates in the regulation of not only humoral responses but also cellular responses, phagocytosis in particular.

It is known that stimulation of TLRs activates multiple signaling pathways, but information about whether other signaling pathways are involved in TLR-mediated phagocytosis is still limited. In this study, we have examined whether TLR-induced phagocytosis is affected by GTPases and actins. Our results suggest that TLRs regulate phagocytosis through a novel actin-Cdc42/Rac pathway.

\section{Results}

Activation of multiple signaling pathways by LPS in RAW264.7 macrophage cells

First, we examined the signaling pathways activated by LPS by stimulating RAW264.7 macrophage cells with LPS for $0,15,30,45,60$, and $120 \mathrm{~min}$. Activation of MAP kinases was measured by anti-phospho MAP kinase antibody. As shown in Figure 1A, stimulation of LPS enhanced the phosphorylation of ERK, JNK, and p38. We assessed the activation of the PI3 kinase signaling pathway by detecting the phosphorylation of Akt, a known downstream target of PI3K, using anti-phospho Akt antibody. LPS stimulation induced phosphorylation of Akt in RAW264.7 macrophages, indicating an activation of PI3K signaling pathways by LPS (Figure 1A). Activation of the NF- $\kappa B$ signaling pathway was analyzed by detecting the expression of IкB, an upstream NF- $\kappa \mathrm{B}$ regulator that binds to NF- $\kappa \mathrm{B}$ and is degraded when the NF- $\kappa \mathrm{B}$ signaling pathway is activated. LPS stimulation induced the degradation of I $\mathrm{B}$, suggesting that the NF- $\kappa B$ signaling pathway is also activated (Figure 1A). To examine the activation of Cdc 42 and Rac1, equal numbers of RAW264.7 macrophage cells were stimulated with LPS, and endogenous GTP-loaded Cdc42 or Rac1 was precipitated from cell lysates using the $\mathrm{Rac} / \mathrm{Cdc} 42$

\section{A}

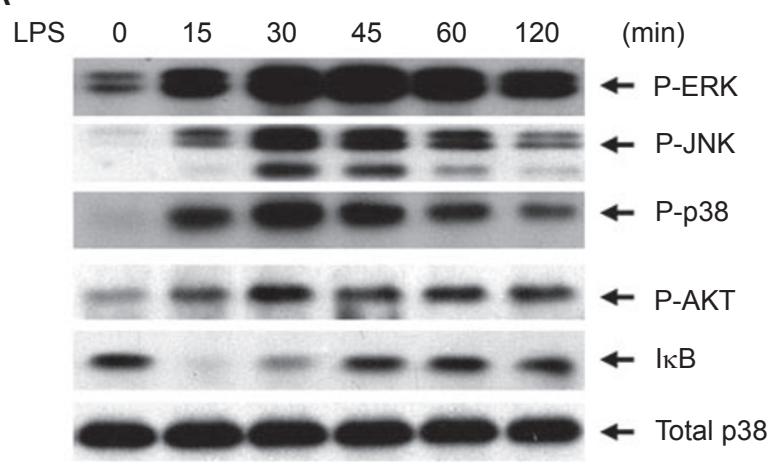

B

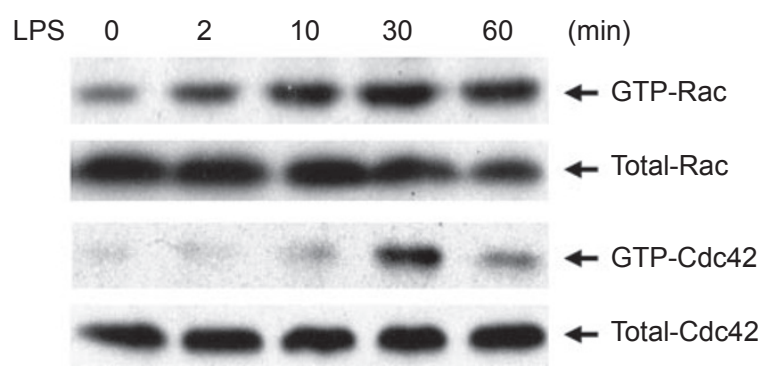

Figure 1 Activation of multiple signaling pathways by LPS in RAW264.7 cells. (A) RAW264.7 macrophage cells were stimulated with LPS $(0.02 \mu \mathrm{g} / \mathrm{ml})$ for the indicated times. Cell lysates from LPS-stimulated RAW264.7 macrophage cells were subjected to western blot analysis by using anti-phospho-ERK, anti-phosphoJNK, anti-phospho-p38, anti-phospho-AKT, and anti-IKB. The amount of p38 noted was used as a loading control. (B) RAW264.7 macrophage cells was treated with LPS and lysed at the time indicated. The GTP-bound forms of endogenous Cdc42 and Rac1 were precipitated by glutathione $S$-transferase (GST)-bound p21-activated kinase 1 (PAK1) (70-106) and detected by western blot analysis using anti-Cdc42 and anti-Rac antibodies. Lysates were also blotted to assess total Cdc42 and Rac1. Data shown are representative of three independent experiments. 
binding fragment of p21-activated kinase 1 (PAK) fused to GST. The challenge of RAW264.7 macrophages from wild-type mice with LPS increased the levels of activated Cdc42-GTP and Rac1-GTP (Figure 1B).

Specific inhibition of the p38 MAP kinase signaling pathway reduced the phagocytosis of GFP-Escherichia coli

Phagocytosis of bacteria is an important feature of the macrophage-mediated innate immune response to bacterial infection [19], which is driven by the reorganization of filamentous actin (F-actin). Recent studies have suggested a linkage of TLR signaling with bacteria phagocytosis [13]. We pretreated RAW264.7 cells with LPS and measured the uptake of the green fluorescent protein (GFP)-labeled strain of $E$. coli in the absence of serum. RAW264.7 cells have a low basal level of phagocytosis of GFP-E. coli as determined by FACS. Stimulation of LPS, a ligand for TLR4, markedly enhanced the phagocytosis of GFP-expressing E. coli as determined by FACs (Figure 2A and 2B). No differences were observed for the phagocytosis of GFP-E. coli at $4{ }^{\circ} \mathrm{C}$, suggesting that internalization, rather than binding, was affected by LPS stimulation (data not shown). LPS pretreatment also enhanced the phagocytosis of FITC-labeled dextran, but not latex beads. To investigate whether activation of MAP kinases plays a role in LPSinduced phagocytosis of GFP-E. coli, RAW264.7 macrophage cells were pretreated with different MAP kinase inhibitors to block the activation of MAP kinase signaling pathways and then measured for their phagocytosis activity. As shown in Figure 2C, neither the JNK-specific inhibitor SP600025 nor the ERK-specific inhibitor PD98059 altered the phagocytosis index. However, specific inhibition of p38 activation by SB203580 reduced LPS-induced phagocytosis of GFP-E. coli (Figure 2C), which is consistent with previous findings [13]. To further confirm the role of $\mathrm{p} 38$ in TLR4-mediated phagocytosis, we inhibited the expression of p38 in RAW264.7 cells by RNAi. Transient transfection of RAW264.7 cells with specific siRNA for p38 markedly reduced the expression of p38 (Figure 2D). Compared with the control group, RAW264.7 cells transfected with p38 siRNA showed attenuated phagocytosis after LPS treatment (Figure 2E).
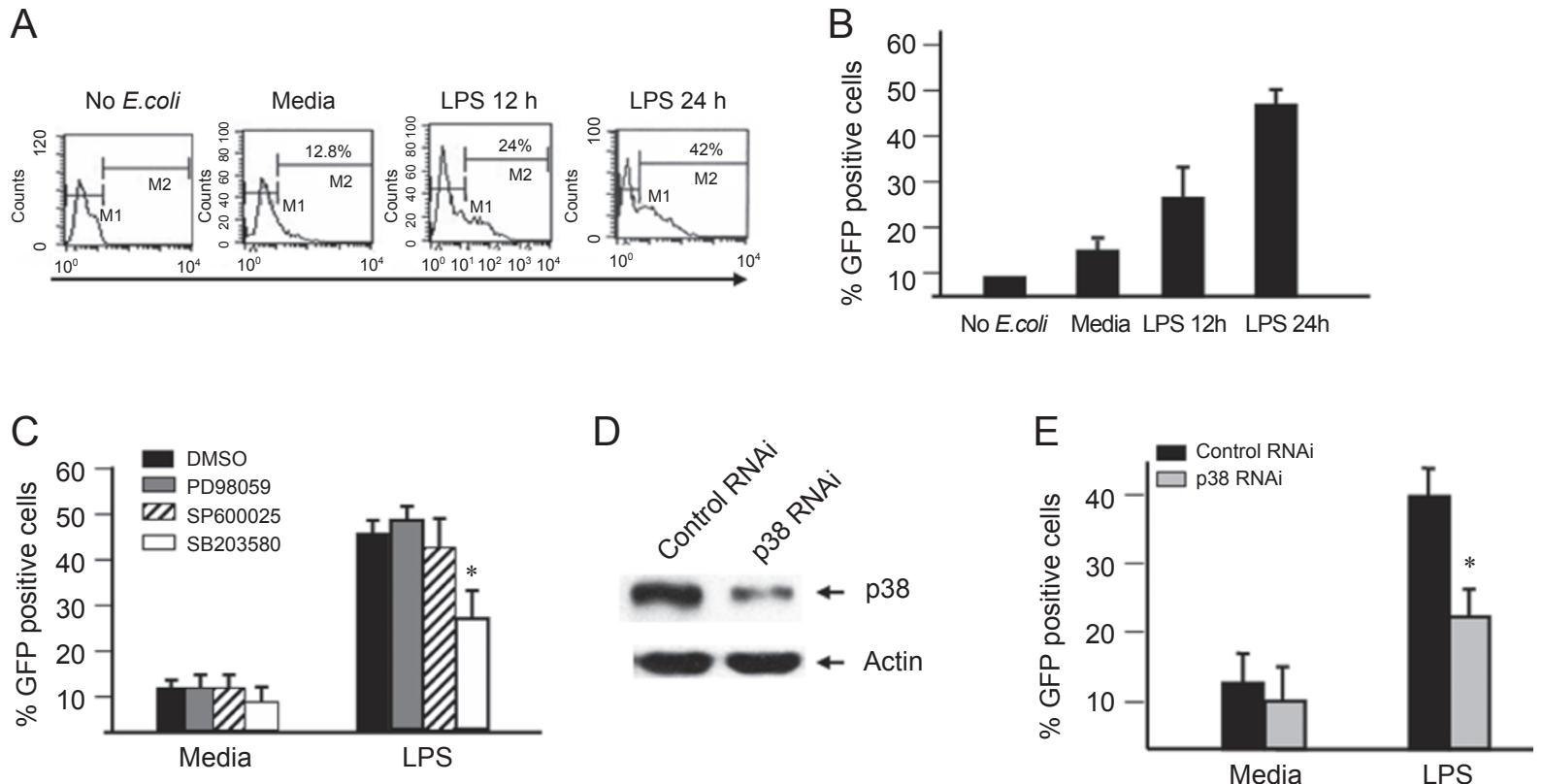

Figure 2 Specific inhibition of the p38 signaling pathway reduced the phagocytosis of GFP-E. coli. (A, B) RAW 264.7 cells were pretreated with media or LPS $(0.02 \mu \mathrm{g} / \mathrm{ml})$ for the times shown, and then incubated with GFP-E. coli at $37^{\circ} \mathrm{C}$ for $45 \mathrm{~min}$. Subsequently, macrophage cells were washed and subjected to FACS ${ }^{\circledR}$ analysis. (C) RAW 264.7 cells were pretreated with

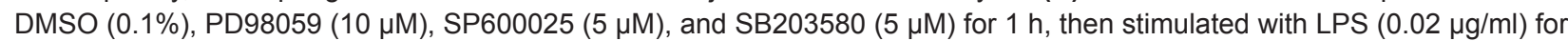
$24 \mathrm{~h}$, and subjected to the phagocytosis experiments with GFP-E. coli. (D) RAW 264.7 cells were transiently transfected with control or p38 siRNA, and the expression level of p38 proteins was detected by western blotting. The amount of actin noted was used as a loading control. (E) Control RNAi or p38 RNAi-transfected RAW264.7 cells were pretreated with media or LPS $(0.02 \mu \mathrm{g} / \mathrm{ml})$ for $24 \mathrm{~h}$ and then infected with GFP-E. coli at $37^{\circ} \mathrm{C}$ for $45 \mathrm{~min}$. Subsequently, macrophage cells were washed and subjected to $\mathrm{FACS}^{\circledR}$ analysis. All FACS ${ }^{\circledR}$ data are represented as percent positive cells. Data shown are the mean \pm SEM of at least three independent experiments. $* P<0.05$. 


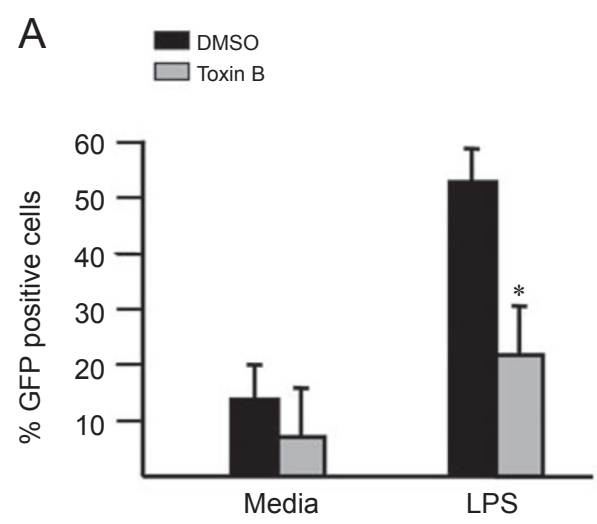

C

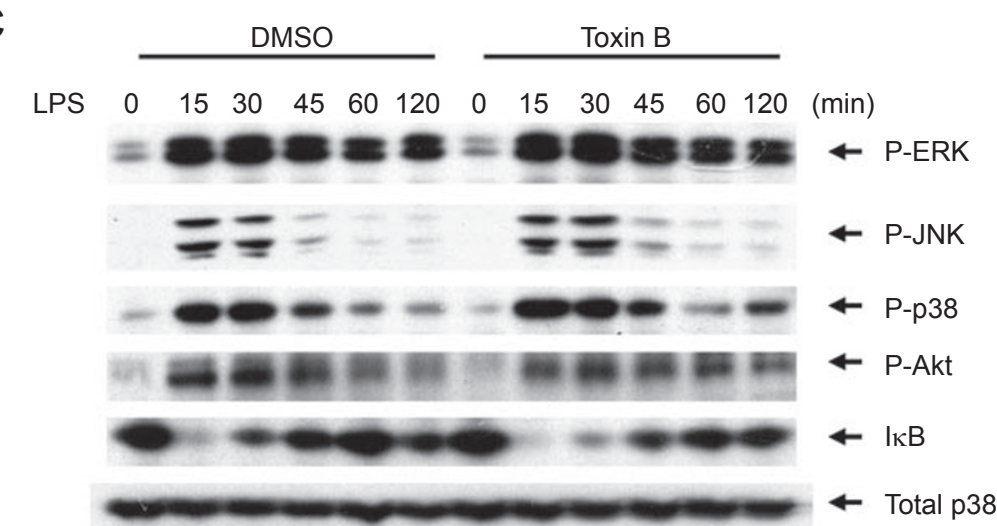

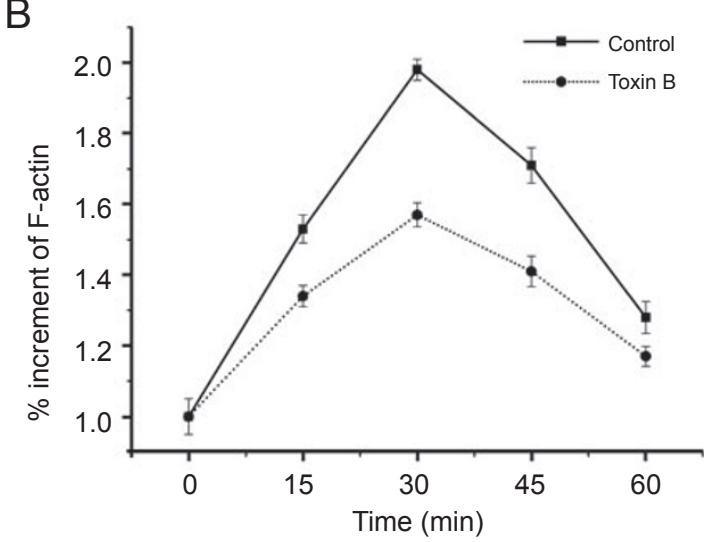

Total p38

Figure 3 Inhibition of the small GTPase signaling pathway by toxin B reduced the phagocytosis of GFP-E. coli. (A) RAW264.7 cells were pretreated with toxin $B(100 \mathrm{ng} / \mathrm{ml})$ for $1 \mathrm{~h}$, stimulated $1 \mathrm{~h}$ later with LPS $(0.02 \mu \mathrm{g} / \mathrm{ml})$ for $24 \mathrm{~h}$, and then subjected to the phagocytosis experiments with GFP-E. coli. Data shown are the mean SEM of three independent experiments. $* P<0.05$. (B) RAW264.7 macrophage cells were pretreated with toxin B or not for $1 \mathrm{~h}$, and then treated with LPS for the indicated time periods. Total F-actin was stained using NBD-phallacidin and measured using an AMINCO Bowman Series 2 spectrofluorometer. F-actin ratio was calculated as (F-actin in activated cells - background)/(F-actin in unstimulated cells - background). (C) Equal numbers of RAW264.7 cells were pretreated with toxin B $(100 \mathrm{ng} / \mathrm{ml})$ for $1 \mathrm{~h}$ and then stimulated with LPS $(0.02 \mu \mathrm{g} / \mathrm{ml})$ for the indicated times. Cell lysates were subjected to western blot analysis by using anti-phospho-ERK, anti-phospho-JNK, anti-phospho-p38, anti-phospho-Akt, and anti-lкB. The amount of p38 noted was used as a loading control. Data shown are representative of three independent experiments.

Inhibition of the small GTPase signaling pathway by toxin $B$ reduced the phagocytosis of GFP-E. coli

Activation of Cdc42 and Rac1 promotes actin polymerization and is essential for macrophage phagocytosis $[3,4]$. To examine whether the small GTPases Cdc42 and Rac1 play a role in TLR4-enhanced phagocytosis, we used Clostridium difficile toxin $\mathrm{B}$, an inhibitor of the small GTPases Rho, Rac, and Cdc42, to inhibit the LPS-stimulated activation of small GTPases. After pretreatment with toxin B for $1 \mathrm{~h}$, RAW264.7 cells were stimulated with LPS for $24 \mathrm{~h}$ and were then subjected to the phagocytosis analysis of GFP- $E$. coli. As shown in Figure 3A, treatment with toxin B markedly reduced both basal and LPS-induced phagocytosis of E. coli. Interestingly, the basal level of phagocytosis was significantly decreased in toxin B-treated RAW264.7 cells compared with controls. To determine whether the inhibition of GTPases in RAW264.7 cells resulted in a failure of actin to polymerize, we quantified $\mathrm{F}$-actin content in cells that were challenged with LPS in the presence or absence of toxin B. In vehicle-treated cells, there was an increase in the total amount of F-actin present after the addition of LPS (Figure 3B), which was blocked by the presence of $2 \mu \mathrm{M}$ cytochalasin D (data not shown). The presence of toxin B inhibited the enhancement of F-actin accumulation by LPS (Figure 3B). These data suggest that inhibition of GTPases may impair actin assembly in response to LPS stimulation. We have also examined whether toxin B regulates the activation of other TLR4 signaling pathways in macrophages after LPS stimulation. As shown in Figure 3C, we found no detectable difference in phospho-ERK, phospho-JNK, 
phospho-p38, or IкB expression between RAW267.4 cells pretreated with toxin $B$ and the control cells, suggesting that small GTPases have no effect on LPS-induced activation of MAPK and NF-KB.

Knockdown of small GTPase expression by RNAi also reduced the phagocytosis of GFP-E. coli

To further examine whether the activation of small GTPases is specifically required for the TLR4-mediated phagocytosis of bacteria, RAW264.7 macrophage cells were transfected with siRNAs specific for Cdc 42 or Rac1. Transfection of Cdc42 siRNAs or Rac1 siRNAs markedly reduced the expression of $\mathrm{Cdc} 42$ and Rac1, respectively
(Figure 4A). RAW264.7 cells transfected with siRNAs targeting Rac1 or Cdc42 showed markedly decreased phagocytosis of GFP-E. coli (Figure 4B). To determine whether Cdc42 or Rac1 affect the phagocytosis of other targets, we performed phagocytosis experiments using FITC-labeled dextran or fluorescently labeled beads. Figure $4 \mathrm{C}$ shows that LPS pretreatment results in a significant increase in the phagocytosis of dextran by macrophage cells as compared with media-pretreated cells, and that RNAi against $\mathrm{Cdc} 42$ or Rac1 markedly reduced both the basal level and LPS-enhanced phagocytosis of dextran. Consistent with a previous study [13], the phagocytosis of latex beads by LPS-treated macrophages was relatively similar
A

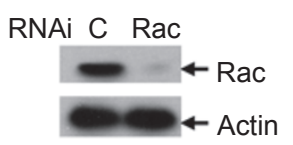

RNAi C Cdc42

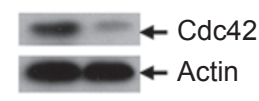

B

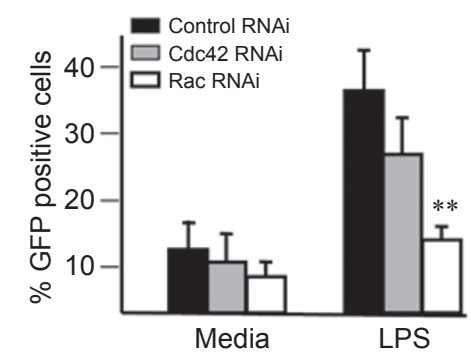

E

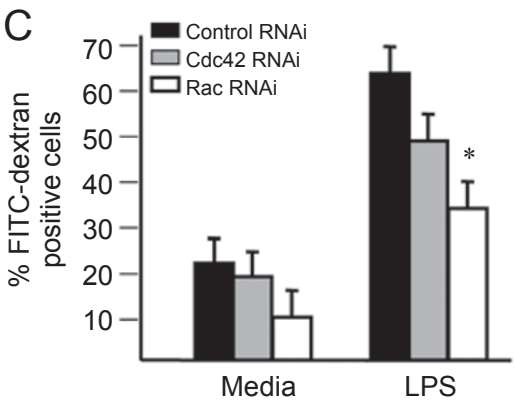

$\mathrm{F}$

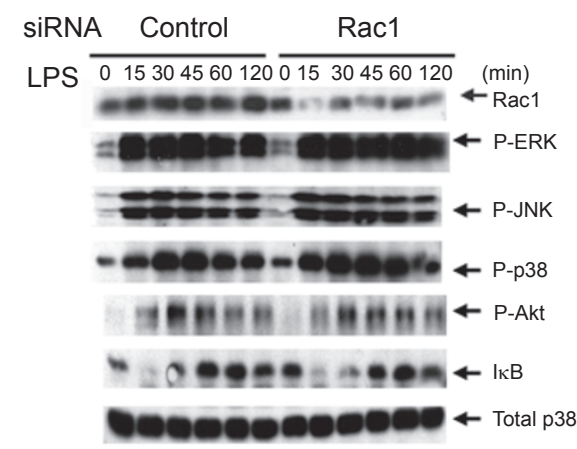

Figure 4 Knockdown of small GTPase expression by RNAi reduced the phagocytosis of GFP-E. coli. (A) RAW264.7 cells were transiently transfected with control, Rac1 siRNA, or Cdc42 siRNA, and all these protein expression levels were detected by western blotting. The amount of actin noted was used as a loading control. (B) RAW264.7 cells transfected with Rac1, Cdc42, or control siRNA were treated with media or LPS $(0.02 \mu \mathrm{g} / \mathrm{ml})$ for $24 \mathrm{~h}$ and then infected with GFP-E. coli at $37^{\circ} \mathrm{C}$ for $45 \mathrm{~min}$. Subsequently, macrophage cells were washed and subjected to FACS ${ }^{\circledR}$ analysis. (C) RAW264.7 cells with Rac1 knockdown, Cdc42 knockdown, or control siRNA were treated with media or LPS $(0.02 \mu \mathrm{g} / \mathrm{ml})$ for $24 \mathrm{~h}$. Cells were then challenged with FITC-dextran at $37^{\circ} \mathrm{C}$ for 45 min and subjected to FACS ${ }^{\circledR}$ analysis. (D) Rac1 siRNA, Cdc42 siRNA,or control siRNA-treated RAW264.7 cells were incubated with media or LPS $(0.02 \mu \mathrm{g} / \mathrm{ml})$ for $24 \mathrm{~h}$. Cells were then challenged with FITC-latex beads at 37 ${ }^{\circ} \mathrm{C}$ for $45 \mathrm{~min}$ and subjected to FACS ${ }^{\circledR}$ analysis. (E) Rac1 knockdown, Cdc42 knockdown, or control siRNA RAW264.7 cells were treated with media or LPS for the indicated time periods. Total F-actin was stained using NBD-phallacidin and measured using an AMINCO Bowman Series 2 spectrofluorometer. F-actin ratio was calculated as (F-actin in activated cells - background)/(Factin in unstimulated cells - background). (F) RAW264.7 cells were transiently transfected with control or Rac1 siRNA. After $48 \mathrm{~h}$ incubation at $37^{\circ} \mathrm{C}$, equal numbers of RAW264.7 cells were treated with LPS $(0.02 \mu \mathrm{g} / \mathrm{ml})$ for the indicated time. Cell lysates were subjected to western blot analysis by using anti-Rac1, anti-phospho-ERK, anti-phospho-JNK, anti-phospho-p38, anti-phospho-AKT, and anti-IкB. The amount of p38 noted was used as a loading control. All FACS ${ }^{\circledR}$ data are represented as percent positive cells, and data shown are the mean SEM of at least three independent experiments. $* P<0.05 ; * * P<0.01$. Other data shown above are representative of at least three independent experiments. 
to that of cells treated with media (Figure 4D). However, we have observed a basal decrease in the phagocytosis of latex beads by $\mathrm{Cdc} 42$ or Rac1 RNAi-treated macrophages. Similarly, LPS-induced actin polymerization was much lower in RAW264.7 cells transfected with Rac1 RNAi or Cdc42-RNAi than in control RNAis (Figure 4E). Activation of ERK, JNK, p38, IкB, and Akt in Rac1 RNAi-treated macrophages showed no detectable difference compared with cells transfected with control siRNA (Figure 4F). Collectively, our data indicate that the small GTPases Rac1 and Cdc42 regulate LPS-induced phagocytosis.

Small GTPases Cdc42 and Racl play an essential role in various TLRs-induced phagocytosis

It has been shown that numerous TLR ligands specifically enhance the phagocytosis of bacteria such as E. coli and Staphylococcus aureus [13]. To examine whether GTPases play a general role in other TLRs-induced phagocytosis,
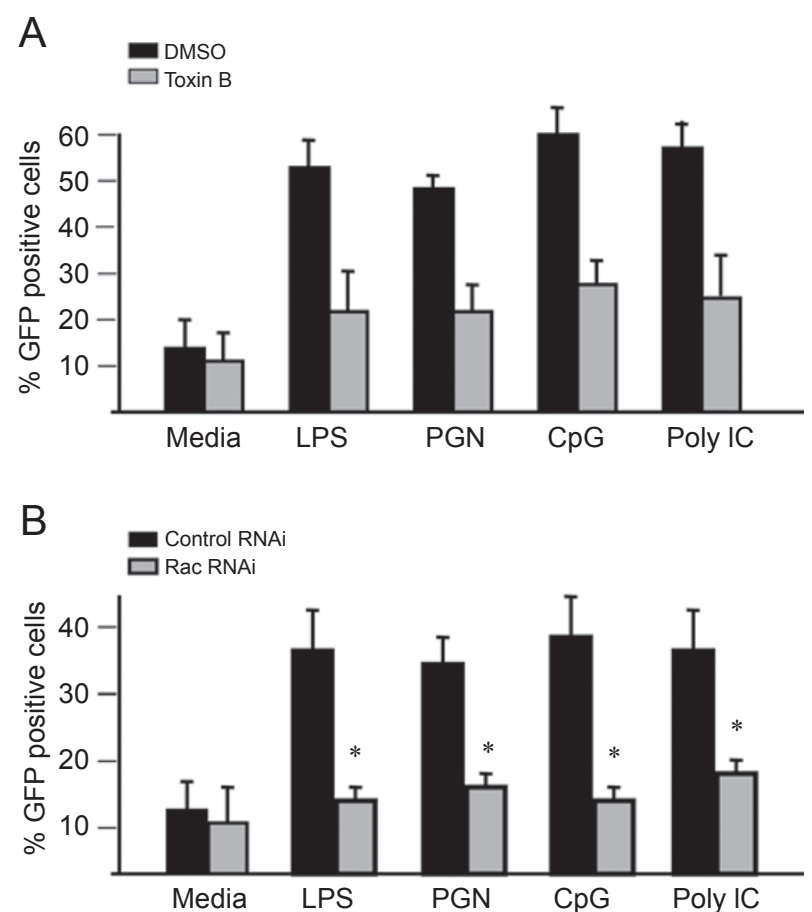

Figure 5 Inhibition of small GTPase signaling pathways reduced other TLR stimuli-induced phagocytosis of GFP-E. coli. (A) RAW264.7 cells were pretreated with toxin $B(100 \mathrm{ng} / \mathrm{ml})$ for $1 \mathrm{~h}$. Cells were then stimulated with LPS $(0.02 \mu \mathrm{g} / \mathrm{ml})$, PGN $(20 \mu \mathrm{g} / \mathrm{ml})$, CpG (100 nM) or polyl:C (10 $\mu \mathrm{g} / \mathrm{ml})$ for $24 \mathrm{~h}$ and subjected to the phagocytosis experiments with GFP-E. coli. (B) RAW264.7 cells were transfected with Rac1 RNAi or with control RNAi. Cells were then stimulated with LPS $(0.02 \mu \mathrm{g} / \mathrm{ml})$, PGN $(20 \mu \mathrm{g} / \mathrm{ml}), \mathrm{CpG}(100$ $\mathrm{nM})$, or polyl:C $(10 \mu \mathrm{g} / \mathrm{ml})$ for $24 \mathrm{~h}$ and subjected to the phagocytosis experiments with GFP-E. coli. All FACS ${ }^{\circledR}$ data are represented as percent positive cells, and data shown are the mean \pm SEM of at least three independent experiments. $* P<0.05$.
RAW264.7 macrophage cells were treated with toxin B to inhibit GTPase activation, and then stimulated with other TLR ligands: purified peptidoglycan (PGN), CpG, and polyinosinic acid (PolyI):C. Similar to LPS, stimulation with either PGN, $\mathrm{CpG}$, or PolyI:C markedly enhanced phagocytosis of GFP-E. coli (Figure 5A, [13]). However, inhibition of GTPase activation by treatment with toxin $\mathrm{B}$ significantly inhibited the enhanced phagocytosis of GFP-E. coli by PGN, CpG or PolyI:C (Figure 5A). Similarly, PGN, CpG, and PolyI:C-induced phagocytosis was substantially reduced in RAW264.7 cells transfected with either Cdc42 RNAi or Rac1 RNAi (Figure 5B), suggesting that the small GTPases Cdc42 and Rac1 play a general role in the regulation of TLR-mediated phagocytosis.

\section{Actin regulates TLR4-stimulated activation of $\mathrm{Cdc} 42$ and} Rac and phagocytosis

It has been shown that phagocytosis is driven by dynamic reorganization of the actin cytoskeleton [20], and organized actin remodeling is critically regulated by the Rho family of small GTPases, i.e. Rac and Cdc42 [21]. As expected, treatment of RAW264.7 cells with cytochalasin $\mathrm{D}$, an actin polymerization inhibitor, markedly reduced LPS-stimulated phagocytosis (Figure 6A). Treatment with microtubule-disrupting agents such as colchicine and nocodazole showed no significant effect on LPS-stimulated phagocytosis. Interestingly, treatment with cytochalasin D also reduced LPS-induced activation of Rac1 and Cdc42 in RAW264.7 cells (Figure 6B). To further explore whether actin plays a specific role in TLR4-induced activation of small GTPases, we transfected RAW264.7 cells with siRNA specific for actin, where the result was a marked reduction in the expression of actin (Figure 6C). RAW264.7 macrophage cells transfected with actin RNAi showed a substantial reduction in the phagocytosis of GFP-E. coli, dextran, and latex beads (Figure 6D). Activation of Cdc42 or Rac1 in actin RNAi-treated RAW264.7 macrophage cells by LPS was much lower than that of cells transfected with control RNAi (Figure 6E). These findings suggest that actin regulates LPS-stimulated activation of $\mathrm{Cdc} 42$ and Rac GTPase.

MyD88 is not required for the activation of the GTPase signaling pathway by LPS

A previous study showed that the basal level of phagocytosis of GFP-E. coli is impaired in MyD88-deficient bone marrow macrophages (BMMs) [13]. CpG-induced phagocytosis of GFP-E. coli is also significantly decreased in

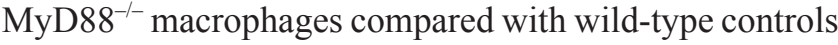
[13]. Consistent with this, our experiments demonstrated that MyD88-deficient primary peritoneal macrophages show less phagocytosis of GFP-E. coli, both at the basal 

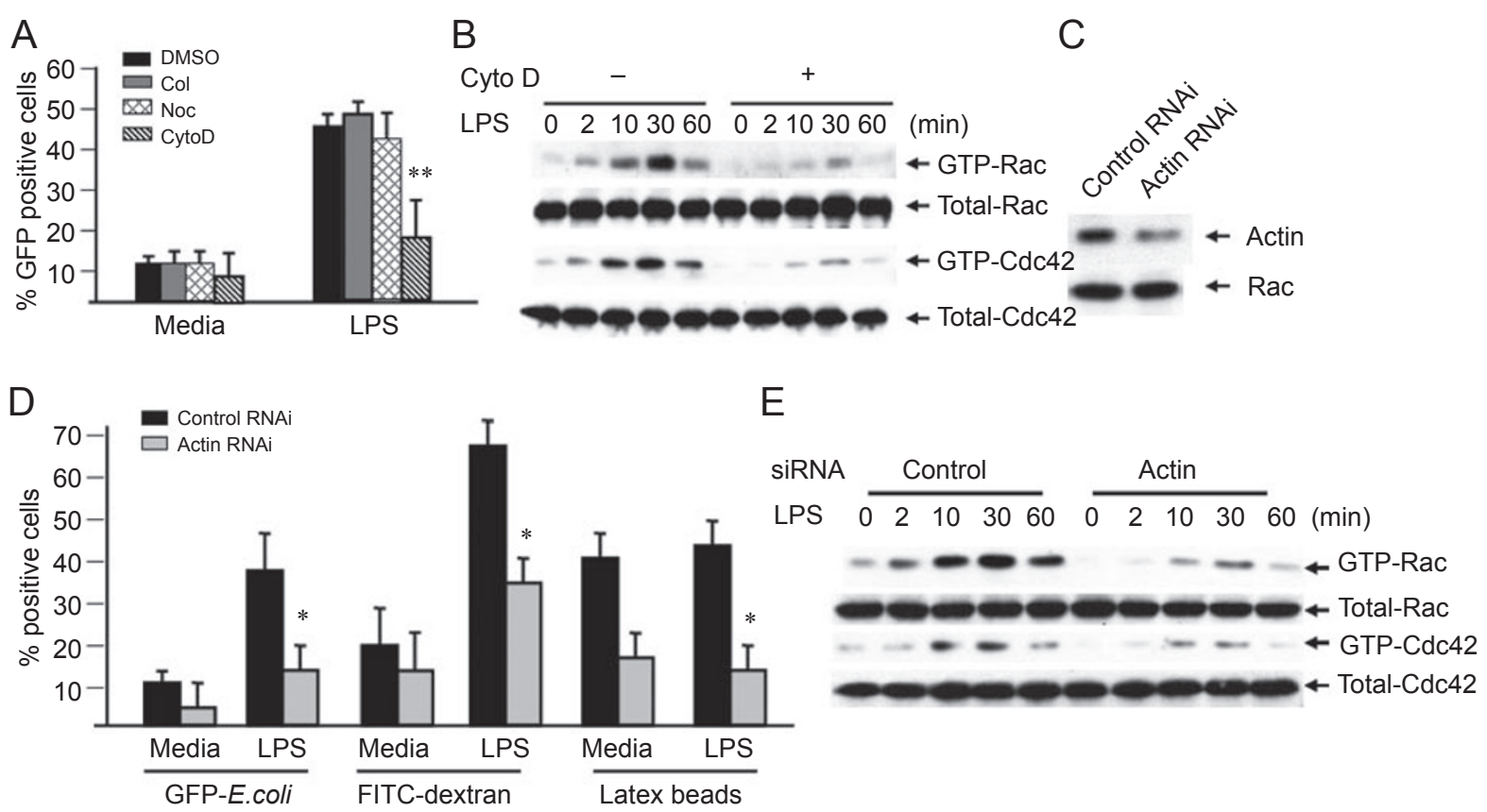

Figure 6 Actin is essential for the activation of GTPases signaling pathway by LPS. (A) RAW264.7 cells were pretreated with DMSO $(0.1 \%)$, colchicine $(5 \mu \mathrm{g} / \mathrm{ml})$, nocodazole $(5 \mu \mathrm{g} / \mathrm{ml})$, and cytochalasin $\mathrm{D}(5 \mu \mathrm{g} / \mathrm{ml})$ for $1 \mathrm{~h}$. Cells were then treated with LPS $(0.02 \mu \mathrm{g} / \mathrm{ml})$ for $24 \mathrm{~h}$ and subjected to the phagocytosis experiments with GFP-E. coli. (B) RAW264.7 cells were pretreated with cytochalasin $D(5 \mu \mathrm{g} / \mathrm{ml})$ for $1 \mathrm{~h}$ and then stimulated with LPS $(0.02 \mu \mathrm{g} / \mathrm{ml})$ for the indicated times. The GTP-bound forms of endogenous Cdc42 and Rac1 were precipitated by glutathione S-transferase (GST)-bound p21-activated kinase 1 (PAK1) (70-106) and detected by western blot analysis using anti-Cdc42 and anti-Rac1 antibodies. Lysates were also blotted to assess total Cdc42 and Rac1. (C) RAW264.7 macrophage cells were transfected with actin or control RNAi. Actin expression levels were detected by western blotting. The amount of Rac1 noted was used as a loading control. (D) Actin knockdown or control RAW264.7 cells were treated with media or LPS $(0.02 \mu \mathrm{g} / \mathrm{ml})$ for $24 \mathrm{~h}$ and then challenged with GFP-E. coli, FITC-dextran, and FITC-latex beads. After incubation at $37{ }^{\circ} \mathrm{C}$ for $45 \mathrm{~min}$, RAW cells were washed and subjected to FACS ${ }^{\circledR}$ analysis. All FACS ${ }^{\circledR}$ data are represented as percent positive cells. Data shown are the mean \pm SEM of five independent experiments. (E) RAW264.7 macrophage cells transfected with actin or with control RNAi were treated with LPS $(0.02 \mu \mathrm{g} / \mathrm{ml})$ for indicated times, and GTPase activation assay was performed as described above. All FACS ${ }^{\circledR}$ data are represented as percent positive cells, and data shown are the mean \pm SEM of at least three independent experiments. $* P<0.05$; $* * P<0.01$. Other data shown above are representative of at least three independent experiments.

level and in the presence of LPS stimulation (Figure 7A). To investigate whether MyD88 is an upstream regulator of small GTPase activation, we stimulated peritoneal macrophages from MyD88 knockout or wild-type mice with LPS and analyzed the activation of Cdc42 and Rac1. Stimulation of LPS caused no detectable difference in the activation of GTP-Rac and GTP-Cdc42 amongst the cells examined (Figure 7B), suggesting that MyD88 is not required for LPS-induced activation of the small GTPases Cdc42 and Rac1.

\section{Discussion}

TLRs are well known as sensors of infectious nonself and as receptors for the activation of the innate immune system [7]. Ligation of TLRs can elicit activation of conserved inflammatory pathways, culminating in the activation of the MAP kinase signaling pathways and IKK complexes, which transduce various upstream signals to the activation of AP-1 and NF- $\mathrm{KB}$ transcription factors [22]. Recent accumulating evidence has shown that TLRs are also involved in phagocytosis by helping phagocytes sense bacteria or their components [23, 24]. Consistent with that, we have provided evidence to show that ligation of TLR 4 can enhance phagocytosis of gram-negative bacteria $E$. coli and nonbacterial targets such as dextran, while exhibiting minimal effects on nonbacterial targets such as latex beads.

Ligation of TLR4 triggers the activation of multiple signaling pathways including p 38 , JNK, ERK, PI3K, and GTPases. Several studies have suggested that the MAPK family of proteins has a critical role in modulating phagocy- 

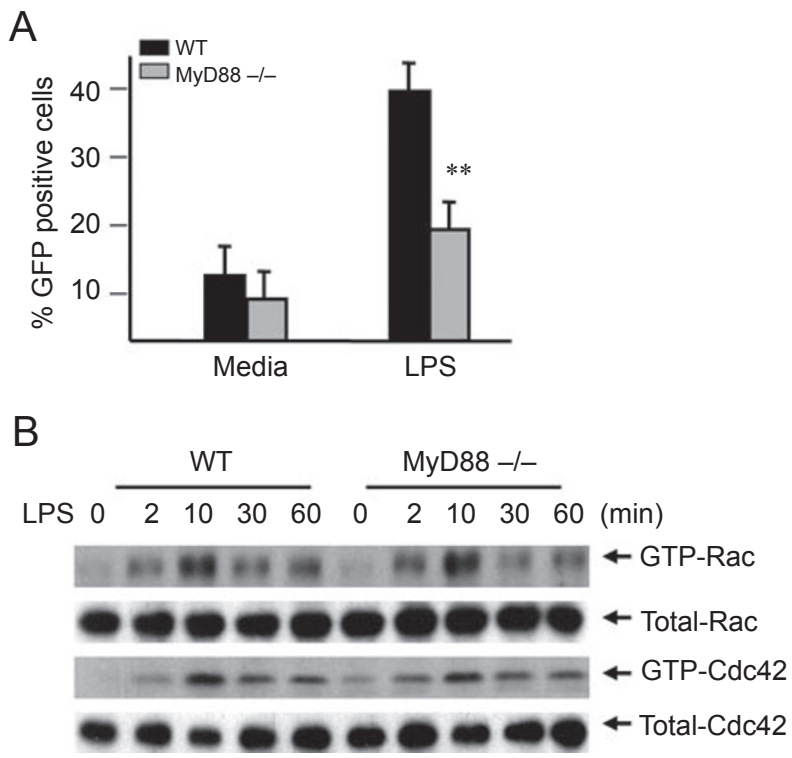

Figure 7 MyD88 is not required for the activation of GTPase signaling pathway by LPS. (A) Peritoneal macrophages from MyD88 knockout or wild-type mice were treated with media or LPS $(0.02$ $\mu \mathrm{g} / \mathrm{ml}$ ) for $24 \mathrm{~h}$ and then challenged with GFP-E. coli. After incubation at $37^{\circ} \mathrm{C}$ for $45 \mathrm{~min}$, macrophage cells were washed and subjected to $\mathrm{FACS}^{\circledR}$ analysis. All FACS ${ }^{\circledR}$ data are represented as percent positive cells. Data shown are the mean \pm SEM of three independent experiments. $* * P<0.01$. (B) Primary peritoneal macrophages from MyD88 knockout or wild-type mice were treated with LPS $(0.02 \mu \mathrm{g} / \mathrm{ml})$ for indicated times, and a GTPase activation assay was performed as described above. Data shown are representative of three independent experiments.

tosis and chemotaxis [25-27]. A previous study even shows that $\mathrm{CpG}$ regulates the phagocytosis of bacteria through p38 [13]. However, the effects of MAPKs on LPS-induced phagocytosis in macrophages have not been characterized in detail. Our results have shown that selective inhibition of p38 activation by a specific inhibitor, or by knocking down $\mathrm{p} 38$ expression using siRNAs, reduced LPS-induced phagocytosis of $E$. coli, suggesting that LPS also regulates phagocytosis of E. coli partially through the MyD88-p38 signaling pathway. PI3K has been shown to provide a critical signal for cell phagocytosis [28, 29]. However, in our experiments, phagocytosis of $E$. coli cannot be blocked by either the PI3K specific inhibitor LY294002 or wortmannin (data not shown). Also, phagocytosis assay of peritoneal macrophages isolated from $\mathrm{p} 110$ mutant and wild-type mice showed no difference in phagocytosis between $\mathrm{p} 110$ mutant and control macrophages (data not shown).

The small GTPases Cdc42 and Rac1 are widely known as critical regulators of the actin cytoskeletal rearrangements that are required for phagocytosis $[3,4]$. Analysis of signaling pathways revealed that $\mathrm{Cdc} 42$ and Rac1 signaling pathways are activated in response to LPS stimulation. Inhibition of Cdc42 and Rac1 activation by $C$. difficile toxin $\mathrm{B}$, or by knocking down Rac1 and Cdc42 expression by RNAi, attenuated actin polymerization and subsequently reduced both the basal level and TLR-enhanced phagocytosis. Our results suggest that small GTPases play an essential role in TLR-mediated phagocytosis. Rac1 has been reported to be essential for LPS-stimulated activation of ERK and p38 kinase in Rat-2 fibroblasts [30], and GTPase is required for BCR-induced JNK and p38 responses [31]. However, another group reported that the small GTPases Cdc42 and Rac are not required for JNK activation by LPS [32]. We demonstrate that, in RAW264.7 cells, knocking down the expression of Rac 1 and $\mathrm{Cdc} 42$ or using a GTPase inhibitor has no effect on p38 activation induced by LPS. Moreover, we examined the LPS-induced GTPase activation in peritoneal macrophages isolated from MyD88 knockout and wild-type mice, and our data showed that there is no difference in LPS-induced activation of Cdc42 and Rac1 between MyD88 knockout and wild-type macrophages. Thus, it is likely that LPS induces the activation of MAPKs and GTPase in macrophages by two distinct pathways.

Members of the Rho subfamily of GTP-binding proteins, namely Rho, Rac, and Cdc42, have been shown to regulate the actin cytoskeleton in response to a variety of extracellular signals [21, 33]. The precise mechanism by which these GTPases are regulated has not yet been defined, but it has been reported that the intrinsic GTP hydrolysis and the GDP/GTP nucleotide exchange reactions of Rho and other Ras superfamily GTPases require the enzymatic assistance of two families of regulators: GTPase-activating proteins (GAPs) and guanine nucleotide exchange factors (GEFs). Interestingly, we find that actin polymerization, which is known as a downstream event of GTPase activation, could regulate the activation of Rac and Cdc42. Cytochalasin D, an actin polymerization inhibitor, can block LPS-induced activation of Rac and Cdc42, and knocking down actin expression by RNAi also leads to reduced Rac and Cdc42 activation. This is the first observation showing that actin is required for the activation of $\mathrm{Cdc} 42$ and Rac by LPS, suggesting a complex interplay between actin and $\mathrm{Cdc} 42$ and Rac.

The precise mechanism by which the signals from LPS-TLR4 affect the phagocytosis of bacteria has not yet been clearly defined, but it has been reported that initial activation of MyD88-IRAK4-p38 by TLR ligands results in secondary up-regulation of scavenger receptors, which promotes phagocytic clearance of bacteria [13]. In our experiments we have observed that LPS-induced Rac activation occurs within less than $1 \mathrm{~h}$ of LPS stimulation, whereas enhanced phagocytosis of bacteria occurs after 12 


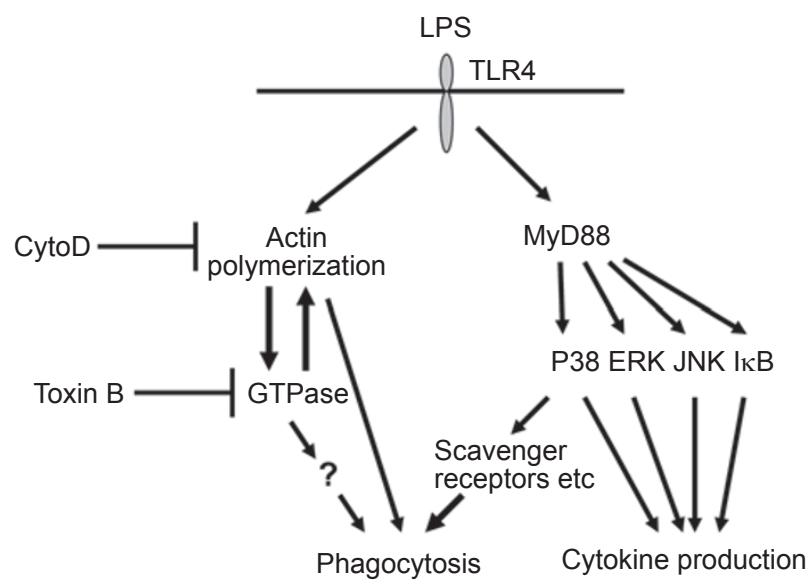

Figure 8 Diagram depicting the hypothesized signaling pathways involved in TLR4-induced immune responses.

or $24 \mathrm{~h}$ of LPS stimulation. Also, LPS-induced activation of Rac and $\mathrm{Cdc} 42$ and the percentage of F-actin returned to basal levels after $1 \mathrm{~h}$. It is likely that the initial activation of Rac, as well as the increase in F-actin by LPS-TLR, may induce a secondary phagocytic gene program that promotes the clearance of bacteria. In summary, we have provided evidence to show that TLRs regulate phagocytosis through the activation of GTPases. Although the MyD88-IRAK4p38 pathway has been reported to regulate phagocytosis, and given the fact that stimulation of TLRs leads to the MyD88-dependent and MyD88-independent activation of multiple signaling pathways, our findings provide an additional mechanism in which the activation of GTPases regulates TLR-mediated phagocytosis through actin assembly, and this pathway is independent of the MyD88p38 signaling pathway. In addition, LPS-induced GTPase activation is regulated by actin polymerization. Thus, it is likely that activation of LPS-TLR4 induces the phagocytic gene program in macrophages through two distinct pathways, one is MyD88-p38-SR (scavenger receptors); another is MyD88-independent activation of the Cdc42 and Rac pathway (Figure 8).

\section{Materials and Methods}

\section{Cell culture and reagents}

The murine macrophage cell line RAW264.7 (RAW) was maintained in Dulbecco's Modified Eagle's Medium supplemented with $10 \%(\mathrm{~V} / \mathrm{V})$ heat-inactivated fetal bovine serum (FBS), $100 \mathrm{U} / \mathrm{ml}$ penicillin $\mathrm{G}$, and $100 \mathrm{U} / \mathrm{ml}$ streptomycin in a humidified $37^{\circ} \mathrm{C}, 5 \%$ $\mathrm{CO}_{2}$ incubator. DMEM and FBS were from GIBCO BRL (Mississauga, ON). Antibodies specific for phosphorylated JNK, p38, and ERK, as well as total p38, were all purchased from Cell Signaling Technology; anti-phospho-IкB antibody was obtained from Santa Cruz Biotechnology. Anti-actin antibody was purchased from Sigma-Aldrich. LPS and PGN were purchased from Sigma-Aldrich; polyinosinic acid (poly I):C was from Amersham Biosciences. The CpG oligodeoxynucleotides 1826 was purchased from InvivoGen. ERK 1/2, JNK, and p38 were inhibited using PD98059, SP600025, and SB203580, respectively (Calbiochem). C. difficile toxin B, cytochalasin D, colchicine, and nocodazole were purchased from Sigma-Aldrich.

\section{Animals and peritoneal macrophages}

$\mathrm{MyD} 88^{--}$mice were obtained from Institut Pasteur, Paris (France), originally from S. Akira (Osaka University, Osaka, Japan), and were backcrossed eight times with $\mathrm{C} 57 \mathrm{BL} / 6$ to ensure similar genetic backgrounds. C57/BL6 mice from which these mice were derived were used as the control mice. These latter mice were supplied by the SLAC laboratory animal center (Shanghai, China). All of the mice were aged between 6 and 8 weeks. Peritoneal macrophages were elicited by i.p. injection of mice with 1-2 $\mathrm{ml}$ thioglycolate (Sigma). The mice were sacrificed 3-4 days later, and the macrophages were harvested by peritoneal lavage, washed twice with $5 \mathrm{ml}$ of PBS, plated in 6-well plates in complete DMEM, and incubated at $37{ }^{\circ} \mathrm{C}$ to allow for adherence. After 2-5 h, non-adherent cells were washed away using PBS, and the remaining cells were incubated in $2 \mathrm{ml}$ of complete DMEM.

\section{Transfection and RNAi}

Cells were transfected using the Amaxa Nucleofector apparatus (Amaxa Biosystems), according to the manufacturer's instructions. Silencing of mouse p38 expression in RAW264.7 cells was achieved by a single p38-specific 21-nucleotide siRNA (Shanghai GenePharma Co., Ltd) corresponding to positions 481-499 (5'-GAC TGT GAG CTC AAG ATT C-3'), and the target sequence of siRNA for mouse $\beta$-actin corresponding to positions $635-653$ is 5'-CAG ACU ACC UCA UGAAGA U-3'. The target sequence of siRNA for mouse Rac1 is 5'-CAC CAC UGU CCC AAC ACU C-3', and for mouse Cde42 is 5'-GAC UCC UUU CUU GCU UGU U-3'; the scrambled negative control siRNA is 5'-UUC UCC GAA CGU GUC ACG U-3'.

\section{Western blot}

Cultured RAW264.7 cells $\left(3-4 \times 10^{6}\right)$ were washed twice with PBS, scraped off the dishes, transferred to a $1.5-\mathrm{ml}$ tube, and centrifuged. The pellet was resuspended in SDS sample buffer and heated at $95{ }^{\circ} \mathrm{C}$ for $10 \mathrm{~min}$. The eluted proteins were applied to SDS-polyacrylamide gels and electrotransferred to nitrocellulose transfer membranes (BioScience). The membranes were blocked for $2 \mathrm{~h}$ in $2 \%$ bovine serum albumin in TBST $(20 \mathrm{mM}$ Tris- $\mathrm{HCl}$ [pH 7.6], $0.15 \mathrm{M}$ sodium chloride, and $0.1 \%$ Tween 20 ), and then incubated for $2 \mathrm{~h}$ with primary antibodies in TBST. After washing three times with TBST, the membranes were incubated for $1 \mathrm{~h}$ with horseradish peroxidase-conjugated anti-mouse or -rabbit immunoglobulin (Amersham Pharmacia Biotech) diluted 1:10 000 in TBST. After three washes in TBST, the blot was developed with the enhanced chemiluminescence system (Amersham Pharmacia Biotech), according to the manufacturer's instructions.

\section{Phagocytosis analysis}

Bacterial phagocytosis by macrophages was examined as described previously [14]. Briefly, the day before commencing the experiment, E. coli strain Top 10 carrying the GFP gene on plasmid Puc-19 was grown from a single colony in Luria-Bertani broth containing $100 \mathrm{~g} / \mathrm{ml}$ ampicillin. Bacteria were then washed in PBS three times and resuspended at the appropriate concentration in DMEM 
media without serum or antibiotics. Before infection, macrophages were washed three times in PBS and then mixed with GFP-E. coli at a multiplicity of infection (MOI) of 10, in DMEM media without serum or antibiotics, at $37^{\circ} \mathrm{C}$ for $45 \mathrm{~min}$. After infection, macrophage cells were washed with cold PBS in order to stop additional bacterial uptake or destruction of bacteria in the phagosome. Cells were washed at least three times in cold PBS, and then fixed using $1 \%$ paraformaldehyde before being harvested in cold PBS containing $5 \mathrm{mM}$ EDTA and subjected to FACS ${ }^{\circledR}$ analysis. Experiments using fluorophore-labeled latex beads (Molecular Probes) were performed in a similar fashion at an MOI of 1. Phagocytosis of dextran was performed as described previously [34]. Briefly, FITC-dextran (Molecular Probes, Eugene, OR) was added to the cell suspension at a final concentration of $1 \mathrm{mg} / \mathrm{ml}$. The cells were incubated for $45 \mathrm{~min}$ at $37^{\circ} \mathrm{C}$, washed with ice-cold PBS, and analyzed using FACS.

Determination of Cdc42 and Racl activation by pull-down assay

The fusion protein GST-CRIB was immobilized by adsorption to glutathione-sepharose beads (Amersham Biosciences, Little Chalfont, UK). After stimulation, cells $\left(1 \times 10^{6}\right)$ were lysed in a buffer containing $25 \mathrm{mM}$ Hepes, pH 7.3, 0.15 M NaCl, $5 \mathrm{mM} \mathrm{MgCl}_{2}, 20$ $\mathrm{mM} 2$-glycerophosphate, $10 \mathrm{mM} \mathrm{NaF}, 2 \mathrm{mM}$ sodium vanadate, $0.5 \%$ Triton X-100, 4\% glycerol, $5 \mathrm{mM}$ dithiothreitol, $0.5 \mathrm{mM}$ phenylmethylsulfonyl fluoride, $5 \mu \mathrm{g} / \mathrm{ml}$ leupeptin, and $5 \mu \mathrm{g} / \mathrm{ml}$ pepstatin. The lysate was clarified by centrifugation, immediately mixed with the immobilized GST-CRIB, and gently rotated at $4{ }^{\circ} \mathrm{C}$ for $60 \mathrm{~min}$. The beads were collected by centrifugation and washed three times with the same buffer. The bound proteins were eluted by heating in SDS-sample buffer and resolved on 12\% SDS-PAGE. Pulled-down GTP-bound forms of Rac and Cdc42 were detected by western blot analysis using anti-Rac and anti-Cdc42 antibodies (Cell Signaling) and visualized using ECL.

\section{Quantitative measurement of F-actin}

The total F-actin content in macrophages was measured as described previously [35]. Briefly, after macrophages were treated with LPS for indicated time periods, the reaction was stopped by the addition of formaldehyde (3.7\% final, V/V) for $15 \mathrm{~min}$ at room temperature. The fixed cells were then permeabilized with $10 \mathrm{mM}$ imidazole, $40 \mathrm{mM} \mathrm{KCl}, 10 \mathrm{mM}$ EGTA, $1 \mathrm{mM} \mathrm{MgCl}_{2}$, and $1 \%$ Triton $\mathrm{X}-100$ at $4{ }^{\circ} \mathrm{C}$ for $15 \mathrm{~min}$. F-actin was then stained with NBD-phallacidin (Molecular Probes) for $2 \mathrm{~h}$ at room temperature. After the cells were washed with PBS, F-actin-bound NBD-phallacidin was extracted with methanol. The extracts were centrifuged to remove any insoluble material, and relative fluorescence was measured using an AMINCO Bowman Series 2 spectrofluorometer (Thermo Spectronic, Madison, Wisconsin, USA) with excitation and emission wavelengths set at 465 and $535 \mathrm{~nm}$, respectively. The F-actin ratio was calculated as (F-actin in activated cells - background)/(F-actin in unstimulated cells - background).

\section{Statistical analysis}

All data are expressed as mean \pm SEM. Student's $t$-test was used for all statistical analysis. Values of $P<0.05$ were considered to be statistically significant.

\section{Acknowledgments}

This work is supported by the National Natural Science Foundation of China (project 30321002 and 30471580), Shanghai E-research Institutes, and Science and Technology Commission of Shanghai Municipality (project 04DZ14902).

\section{References}

1 Greenberg S, Grinstein S. Phagocytosis and innate immunity. Curr Opin Immunol 2002; 14:136-145.

2 Underhill DM, Ozinsky A. phagocytosis of microbes: complexity in action. Annu Rev Immunol 2002; 20:825-852.

3 Caron E, Hall A. Identification of two distinct mechanisms of phagocytosis controlled by different Rho GTPases. Science 1998; 282:1717-1721.

4 Cox D, Chang P, Zhang Q, Reddy PG, Bokoch GM, Greenberg S. Requirements for both Rac1 and Cdc42 in membrane ruffling and phagocytosis in leukocytes. J Exp Med 1997; 186:1487-1494.

5 Boguski MS, McCormick F. Proteins regulating Ras and its relatives. Nature 1993; 366:643-654.

6 Akira S, Takeda K. Toll-like receptor signalling. Nat Rev Immunol 2004; 4:499-511.

7 Medzhitov R. Toll-like receptors and innate immunity. Nat Rev Immunol 2001; 1:135-145

8 Poltorak A, He X, Smirnova I, et al. Defective LPS signaling in $\mathrm{C} 3 \mathrm{H} / \mathrm{HeJ}$ and $\mathrm{C} 57 \mathrm{BL} / 10 \mathrm{ScCr}$ mice: mutations in Tlr4 gene. Science 1998; 282:2085-2088.

9 Ono K, Han J. The p38 signal transduction pathway activation and function. Cell Signal 2000; 12:1-13.

10 Beutler B, Kruys V. Lipopolysaccharide signal transduction, regulation of tumor necrosis factor biosynthesis, and signaling by tumor necrosis factor itself. J Cardiovasc Pharmacol 1995; 25:S1-S8.

11 Ozinsky A, Underhill DM, Fontenot JD, et al. The repertoire for pattern recognition of pathogens by the innate immune system is defined by cooperation between toll-like receptors. Proc Natl Acad Sci USA 2000; 97:13766-13771.

12 Underhill DM, Ozinsky A, Hajjar AM, et al. The toll-like receptor 2 is recruited to macrophage phagosomes and discriminates between pathogens. Nature 1999; 401:811-815.

13 Doyle SE, O'Connell RM, Miranda GA, et al. Toll-like receptors induce a phagocytic gene program through p38. J Exp Med 2004; 199:81-90.

14 Blander JM, Medzhitov R. Regulation of phagosome maturation by signals from toll-like receptors. Science 2004; 304:10141018

15 Yates RM, Russell DG. Phagosome maturation proceeds independently of stimulation of toll-like receptors 2 and 4. Immunity 2005; 23:409-417.

16 Chen K, Iribarren P, Hu J, et al. Activation of Toll-like receptor 2 on microglia promotes cell uptake of Alzheimer disease-associated amyloid beta peptide. J Biol Chem 2006; 281:3651-3659.

17 Luther K, Torosantucci A, Brakhage AA, Heesemann J, Ebel F. Phagocytosis of Aspergillus fumigatus conidia by murine macrophages involves recognition by the dectin-1 beta-glucan receptor and Toll-like receptor 2. Cell Microbiol 2007; 9:368-381.

18 Neal MD, Leaphart C, Levy R, et al. Enterocyte TLR4 mediates phagocytosis and translocation of bacteria across the intestinal 
barrier. J Immunol 2006; 176:3070-3079.

19 Aderem A, Underhill DM. Mechanisms of phagocytosis in macrophages. Annu Rev Immunol 1999; 17:593-623.

20 Greenberg S. Signal transduction of phagocytosis. Trends Cell Biol 1995; 5:93-99.

21 Hall A. Rho GTPase and the actin cytoskeleton. Science 1998; 279:509-514.

22 Dong C, Davis RJ, Flavell RA. MAP kinases in the immune response. Annu Rev Immunol 2002; 20:55-72.

23 Fitzgerald KA, Rowe DC, Golenbock DT. Endotoxin recognition and signal transduction by the TLR4/MD2-complex. Microbes Infect 2004; 6:1361-1367.

24 Qureshi ST, Medzhitov R. Toll-like receptors and their role in experimental models of microbial infection. Genes Immun 2003; 4:87-94.

25 Nick JA, Avdi NJ, Gerwins P, Johnson GL, Worthen GS. Activation of a $\mathrm{p} 38$ mitogen activated protein kinase in human neutrophils by lipopolysaccharide. J Immunol 1996; 156:4867-4875.

26 Schnyder B, Meunier PC, Car BD. Inhibition of kinases impairs neutrophil activation and killing of Staphylococcus aureus. Biochem J 1998; 331:489-495.

27 Downey GP, Butler JR, Tapper H, et al. Importance of MEK in neutrophil microbicidal responsiveness. J Immunol 1998; 160:434-443.

28 Leverrier Y, Okkenhaug K, Sawyer C, Bilancio A, Vanhaesebroeck B, Ridley AJ. Class I phosphoinositide 3-kinase p110beta is required for apoptotic cell and Fcgamma receptor-mediated phagocytosis by macrophages. J Biol Chem 2003; 278:38437-
38442.

29 Allen LA, Allgood JA, Han X, Wittine LM. Phosphoinositide3kinase regulates actin polymerization during delayed phagocytosis of Helicobacter pylori. J Leukoc Biol 2005; 78:220-230.

30 Woo CH, Kim JH. Rac GTPase activity is essential for lipopolysaccharide signaling to extracellular signal-regulated kinase and p38 MAP kinase activation in rat-2 fibroblasts. Mol Cells 2002; 13:470-475.

31 Hashimoto A, Okada H, Jiang A, et al. Involvement of guanosine triphosphatases and phospholipase C-g2 in extracellular signalregulated kinase, c-Jun NH2-terminal kinase, and p38 mitogenactivated protein kinase activation by the $\mathrm{B}$ cell antigen receptor. J Exp Med 1998; 188:1287-1295.

32 Procyk KJ, Rippo MR, Testi R, Hofmann F, Parker PJ, Baccarini M. Lipopolysaccharide induces Jun N-terminal kinase activation in macrophages by a novel Cdc42/Rac-independent pathway involving sequential activation of protein kinase $\mathrm{C} \mathrm{z}$ and phosphatidylcholine-dependent phospholipase C. Blood 2000; 96:2592-2598.

33 Chimini G, Chavrier P. Function of Rho family proteins in actin dynamics during phagocytosis and engulfment. Nat Cell Biol 2000; 2:E191-E196.

34 Gröger M, Holnthoner W, Maurer D, et al. Dermal microvascular endothelial cells express the 180-kDa macrophage mannose receptor in situ and in vitro. J Immunol 2000; 165:5428-5434.

35 Frigeri L, Apgar JR. The role of actin microfilaments in the downregulation of the degranulation response in RBL-2H3 mast cells. J Immunol 1999; 162:2243-2250. 